\title{
Successful medical management of insulinoma with diazoxide for 27 years
}

\author{
Annabelle M Warren', Duncan J Topliss ${ }^{1,2}$ and Peter Shane Hamblin $\mathbb{D D}_{1,2,3,4}$ \\ 1Department of Endocrinology and Diabetes, Alfred Health, Melbourne, Victoria, Australia, 2Monash University, \\ Central Clinical School, Melbourne, Victoria, Australia, ${ }^{3}$ Department of Endocrinology and Diabetes, Western Health, \\ Melbourne, Victoria, Australia, and ${ }^{4}$ Department of Medicine, Western Clinical School, The University of Melbourne, \\ Melbourne, Victoria, Australia
}

Correspondence should be addressed to P S Hamblin

Email

peter.hamblin@wh.org.au

\section{Summary}

Despite improvements in localisation techniques and surgical advances, some patients with insulinoma will not be cured by surgery or may not be suitable for surgery. Medical management with diazoxide is an option for such cases. This case report details 27 years of successful management of insulinoma using diazoxide. It has been effective and safe, with only minor adverse effects.

\section{Learning points:}

- Long term diazoxide use can be a safe, effective option for insulinoma when it cannot be localised or removed surgically.

- Common adverse effects include peripheral oedema, hyperuricaemia, and hirsutism.

- ${ }^{68} \mathrm{Ga}-\mathrm{NOTA}$-exendin-4 PET/CT scan should be considered for insulinoma localisation when other modalities have been unhelpful.

\section{Background}

Despite significant improvements in localisation techniques and surgical management for insulinoma, ongoing medical therapy is sometimes required. Some insulinomas still evade localisation, while for others, surgery may be unsuccessful despite apparent localisation. Some patients decline repeat surgery for insulinoma persistence or recurrence, especially if that involves total pancreatectomy and some are unfit for surgery.

We report the case of an insulinoma successfully managed with oral diazoxide for 27 years. To our knowledge this is the longest reported duration of safe, effective use of diazoxide for the management of insulinoma.

\section{Case presentation}

The patient, a primary school physical education teacher, was diagnosed with insulinoma in October 1992 at the age of 38. Two and a half years earlier, he had developed symptoms including multiple episodes of erratic behaviour, slurred speech, and in retrospect nocturnal generalised tonic-clonic seizures. The episodes typically occurred at night, especially after physical activity during the day. His wife noted that he had frequent episodes of cold, clammy skin with profuse sweating in bed at night. Between episodes, he was described by his wife as increasingly 'vague'. The patient noted that drinking juice or eating carbohydrate helped him feel better at times. He did not experience weight gain in this period.

Despite these symptoms, he was still able to continue working as a primary school physical education teacher and regularly play cricket on weekends during the summer.

He was misdiagnosed by several doctors and thought to have a psychiatric condition. He was admitted on one occasion to an inpatient psychiatry unit after displaying bizarre behaviour when working as a teacher at his school. 
After more than 2 years of assessments and much distress, a psychiatrist eventually performed a random laboratory blood glucose level (BGL), which was 1.7 $\mathrm{mmol} / \mathrm{L}(31 \mathrm{mg} / \mathrm{dL}), 3 \mathrm{~h}$ after a meal.

He was admitted for a $72 \mathrm{~h}$ fast in October 1992. Within $2 \mathrm{~h}$ of the start of the fast, he had a generalised tonic-clonic seizure associated with a low laboratory BGL of $1.8 \mathrm{mmol} / \mathrm{L}$ ( $32 \mathrm{mg} / \mathrm{dL}$ ), inappropriately elevated levels of insulin of $95 \mathrm{mU} / \mathrm{L}$ (normal fasting <10) and C-Peptide of $1.37 \mathrm{pmol} / \mathrm{L}(0.18-0.63)$. A sulfonylurea screen was not performed. CT pancreas was unremarkable with no mass lesions seen. He was discharged on a regimen of four hourly carbohydrate-containing meals (including overnight) pending further investigation. This improved his symptoms significantly.

In November 1992, he underwent the invasive study used at that time for insulinoma localisation, percutaneous transhepatic portal and splenic vein sampling. This identified maximal insulin secretion just distal to the entry of the superior mesenteric vein.

One week later he proceeded to surgery, with open exploration of the pancreas. Inspection and palpation of the pancreas failed to reveal any obvious pancreatic lesion. Distal pancreatectomy was then performed, with the intention of resecting the area identified during transhepatic sampling as most likely to be harbouring an insulinoma. The surgery was complicated by the need for splenectomy and a post-operative collection requiring drainage.

Histology of the resected pancreas was normal with no evidence of insulinoma or nesidioblastosis.

He continued to have symptomatic hypoglycaemia. He was able to avoid severe symptoms with frequent small meals containing carbohydrate, but unfortunately, this led to $17 \mathrm{~kg}$ weight gain over the following 3 months.

Repeat $72 \mathrm{~h}$ fast in February 1993 was terminated after $9 \mathrm{~h}$ because of symptomatic low laboratory BGL of $2.6 \mathrm{mmol} / \mathrm{L}(47 \mathrm{mg} / \mathrm{dL})$ with a paired insulin level of 26 $\mathrm{mU} / \mathrm{L}(<10)$. Repeat imaging with both fine cut CT and MRI pancreas again failed to identify any discrete lesions.

Plasma insulin levels before and after a $50 \mu \mathrm{g}$ octreotide subcutaneous test dose showed apparent transient suppression of insulin, maximal at $2 \mathrm{~h}$ postoctreotide. However, a subsequent Indium ${ }^{111}$ labelled somatostatin nuclear scan was negative.

Repeat percutaneous transhepatic portal vein sampling in April 1993, this time also with selective intraarterial calcium stimulation, demonstrated a marked rise in insulin after gastro-duodenal artery calcium injection, consistent with the presence of an insulinoma with blood supply most closely linked to the distribution of the gastro-duodenal artery.

A subsequent pancreatic endoscopic ultrasound examination was unremarkable.

In July 1993, he underwent repeat open pancreatic surgery. Intraoperative physical and ultrasound examination of the pancreas and duodenum failed to localise a lesion once again. The surgeon decided not to proceed with total pancreatectomy.

Oral diazoxide was then commenced. The dose was progressively increased over the following days to 200 $\mathrm{mg}$ three times daily by the time of discharge. With this dose, he was able to maintain BGLs above $5 \mathrm{mmol} / \mathrm{L}$ $(90 \mathrm{mg} / \mathrm{dL}$ ).

The diazoxide dose was periodically adjusted in the outpatient setting. He had a marked reduction in hypoglycaemic symptoms. He felt very well and returned to full-time work as a physical education teacher.

He experienced adverse effects of mild ankle oedema and hirsutism. He was treated with a thiazide diuretic to manage the peripheral oedema with oral potassium supplementation.

Neuropsychology assessment in 1995 demonstrated a significant improvement in short term memory, functioning, concentration, and general mental acuity compared to his baseline assessment in 1992.

In 2000, he experienced two generalised tonic-clonic seizures in the context of missed doses of diazoxide. At that time, he was supposed to be taking $600 \mathrm{mg}$ diazoxide daily in divided doses. He has not had another seizure since.

He was trialled with subcutaneous Octreotide in addition to diazoxide, but this was discontinued after 3 weeks because of gastrointestinal side effects.

Further imaging with CT and MRI in 2002 again failed to localise a lesion.

In January 2013, he underwent a Ga-68 DOTAoctreotate nuclear scan which was negative.

In July 2014, ${ }^{68} \mathrm{Ga}$-NOTA-exendin-4 PET/CT scan was performed that appeared to localise the lesion. This showed intense uptake associated with an $11 \mathrm{~mm}$ nodule located in the medial aspect of the junction between the pancreatic head and neck, adjacent to the portal vein (Figure 1). The lesion had abnormal fat density, potentially representing fatty hypertrophy due to local paracrine effects of chronic hyperinsulinemia. There was no evidence of regional nodal or metastatic disease. While fasting for the scan, his BGL fell to 


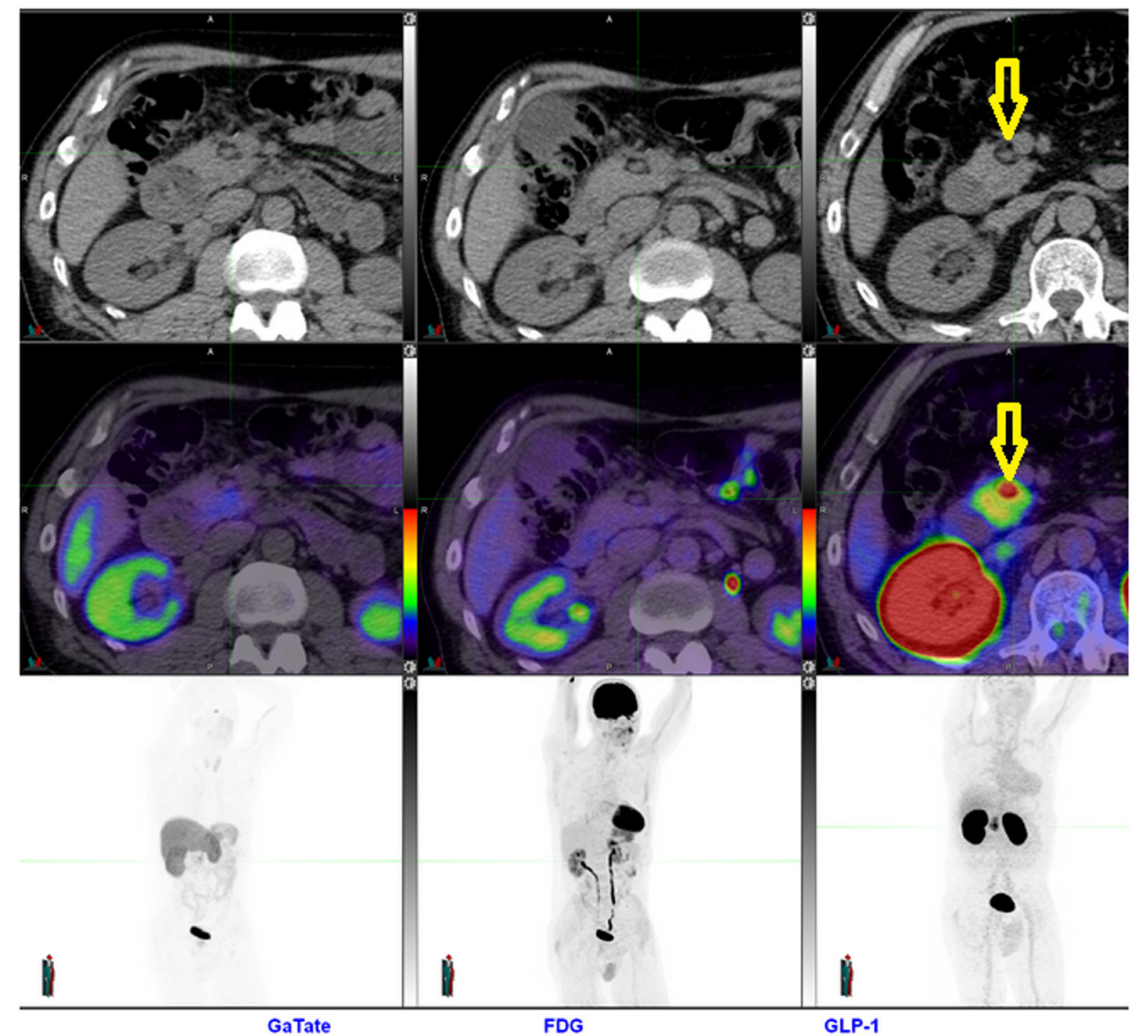

\section{Figure 1}

Apparent localisation of insulinoma (arrowed) with ${ }^{8} \mathrm{Ga}-\mathrm{NOTA}$-exendin-4 PET/CT scan (GLP-1 scan). CT pancreas abnormality previously attributed to post-operative change. Negative ${ }^{68} \mathrm{Ga}$ DOTA-octreotate (GaTate) and FDG PET scans.
$1.8 \mathrm{mmol} / \mathrm{L}(32 \mathrm{mg} / \mathrm{dL})$. Frustratingly, the apparent lesion was located only $5 \mathrm{~mm}$ from the previous surgical resection margin. In retrospect, the lesion was visible on plain CT scan but had been attributed to post-operative change (Figure 1).

Surgical advice was that it was highly likely he would require total pancreatectomy to resect this lesion. His symptoms remained adequately managed on diazoxide (total daily dose of $400 \mathrm{mg}$ at that time) and the patient opted to continue with medical management rather than undergo further surgery, given the expected complications of insulin-requiring diabetes and exocrine pancreatic insufficiency.

In 2017, he undertook 14 days of flash glucose monitoring to evaluate his response to diazoxide therapy. This demonstrated overnight hypoglycaemia on three occasions, with a nadir BGL of $2.2 \mathrm{mmol} / \mathrm{L}$, and one hypoglycaemic episode in the afternoon coinciding with physical activity. All other BGLs were in the normal range.

After further lengthy discussions, the patient again expressed a preference to continue diazoxide therapy rather than have pancreatic surgery. His strong preference was to avoid the need for multiple insulin injections or insulin pump therapy and the attendant risks of hypoglycaemia associated with that and the need for pancreatic exocrine replacement therapy.

\section{Investigation}

October 1992 and February 1993: 72-h fasts with paired low blood glucose, high insulin and high C-peptide levels (terminated at 2 and $9 \mathrm{~h}$, respectively, because of symptomatic hypoglycaemia).

November 1992 and April 1995: Neuropsychology testing showed Memory quotient of 97 in 1992 and 114 in 1995. Measurable improvement in short term memory functioning, concentration, sequencing ability and general mental acuity.

The following imaging modalities were all negative for insulinoma localisation:

\section{2, 1993, 2002: CT pancreas}

1992: Percutaneous transhepatic portal and splenic vein sampling 1993, 2002: MRI pancreas

1993: Repeat percutaneous transhepatic portal vein sampling on this occasion also with selective intraarterial calcium stimulation

1993: Indium ${ }^{111}$ labelled somatostatin nuclear scan

1993: Pancreatic endoscopic ultrasound

2013: Ga-68 DOTA-octreotate nuclear scan

July 2014: Apparent localisation: ${ }^{68} \mathrm{Ga}-\mathrm{NOTA}-\mathrm{exendin}-4$ PET/CT scan 


\section{Treatment}

1992 to current: Regular carbohydrate-containing meals, including a snack at bedtime.

1992: Distal pancreatectomy and splenectomy: normal pancreatic histology.

1993: Repeat open surgery, pancreatic inspection, palpation, and intraoperative ultrasound: insulinoma not identified, no pancreatic surgery undertaken.

July 1993: Diazoxide commenced. Daily doses have varied from 400 to $700 \mathrm{mg}$ (given in divided doses); current dose is $400 \mathrm{mg}$.

2000: Trial of subcutaneous Octreotide - discontinued after 3 weeks because of adverse gastrointestinal effects.

\section{Outcome and follow-up}

He is now aged 66 and has been followed from 1992 to 2020. He was last reviewed in June 2020. He retired from teaching by choice at the age of 55 and subsequently worked on a volunteer basis preparing the wicket for his local cricket ground every season until he moved with his wife 2 years ago to a small coastal town. He remains physically active and has not had any hypoglycaemic episodes requiring the assistance of others apart from the two episodes when he missed diazoxide doses in 2000.

He does, however, have mild hypoglycaemic symptoms several times a month and his wife reported that she feels he has been becoming more forgetful over the last couple of years. His mother had early onset dementia in her late fifties and his wife is concerned that he may be starting to experience the early symptoms of this. He has yet to be formally evaluated for this possibility. It is also possible that the many years of intermittent hypoglycaemia may be contributing to this.

His weight has fluctuated from $80 \mathrm{~kg}$ in 1992 with a peak of $97 \mathrm{~kg}$ in 2014. Most recent weight in June 2020 was $89.9 \mathrm{~kg}$ with BMI of $26.9 \mathrm{~kg} / \mathrm{m}^{2}$.

Apart from mild peripheral oedema controlled with thiazide diuretics, he has developed gout with elevated uric acid. This may be an adverse effect of either diazoxide or the thiazide diuretic. He has developed generalised hirsutism which does not worry him. His routine renal function, liver function, lipids and haematology tests have all remained normal.

\section{Discussion}

Diazoxide, a non-diuretic benzothiadiazide, primarily elevates blood glucose by inhibiting insulin secretion from pancreatic beta cells and also increases hepatic glucose production (1). The main adverse effects are fluid retention, hirsutism, nausea, elevated uric acid levels and thrombocytopenia (FDA Product Information https://www.accessdata.fda.gov/drugsatfda_docs/ label/2015/017453s020lbl.pdf, accessed 13 July 2020).

To our knowledge, this is the longest reported use of diazoxide for insulinoma treatment. We identified a report of long term successful management of insulinoma with diazoxide for 11 years (2) and a UK survey of 40 diazoxide treated insulinoma patients reported a mean duration of treatment of 6 years, with a range up to 22 years (3).

This case is an interesting historical study of the progress that has been made in the investigation of insulinomas over the past three decades. It is worth noting the potential usefulness of ${ }^{68} \mathrm{Ga}$-NOTA-exendin-4 PET/CT scanning (4) for cases where traditional imaging fails to localise an insulinoma. This was the only modality that was effective in our case.

Although we have recommended further surgery now that we believe we have localised the insulinoma, the patient's quality of life obtained from the long term use of diazoxide is sufficiently good that he has chosen to continue medical management rather than require insulin and pancreatic enzyme support after likely total pancreatectomy. Endoscopic ultrasound (EUS)-guided ethanol ablation of the lesion is an alternative potential strategy that is reported to be effective (5), but currently, the patient does not wish to pursue this intervention.

Despite medical advances, there will remain patients who are not suitable surgical candidates, where insulinoma localisation has failed or where a patient declines intervention. Long term medical management with diazoxide may achieve significant improvement in hypoglycaemic symptom burden and quality of life, with tolerable adverse effects.

Declaration of interest

The authors declare that there is no conflict of interest that could be perceived as prejudicing the impartiality of the research reported.

\section{Funding}

This research did not receive any specific grant from any funding agency in the public, commercial or not-for-profit sector.

Patient consent

Written informed consent has been obtained from the patient for publication of the submitted article and accompanying images. 


\section{Author contribution statement}

A M W prepared the first draft and contributed to case report revisions. D J $T$ involved in patient management decisions over entire period; interpreted endocrine testing results; contributed to case report revisions. $\mathrm{P} \mathrm{S} \mathrm{H}$ is a physician responsible for patient for entire period, contributed to case report revisions.

\section{References}

1 Altszuler N, Moraru E \& Hampshire J. On the mechanism of diazoxide-induced hyperglycemia. Diabetes 197726 931-935. (https://doi.org/10.2337/diab.26.10.931)
2 Goode PN, Farndon JR, Anderson J, Johnston IDA \& Morte JA. Diazoxide in the management of patients with insulinoma. World Journal of Surgery 198610 586-592. (https://doi.org/10.1007/ BF01655532)

3 Gill GV, Rauf O \& MacFarlane IA. Diazoxide treatment for insulinoma: a national UK survey. Postgraduate Medical Journal 1997 73 640-641. (https://doi.org/10.1136/pgmj.73.864.640)

4 Pattison DA \& Hicks RJ Molecular imaging in the investigation of hypoglycaemic syndromes and their management. Endocrine-Related Cancer 201724 R203-R221. (https://doi.org/10.1530/ERC-17-0005)

5 Levy MJ, Thompson GB, Topazian MD, Callstrom MR, Grant CS \& Vella A. US-guided ethanol ablation of insulinomas: a new treatment option. Gastrointestinal Endoscopy 201275 200-206. (https://doi. org/10.1016/j.gie.2011.09.019)

Received in final fo 27 August 2020

Accepted 10 September 2020 